\title{
Sur la nasale palatale et les rimes approximatives en anglo-normand
}

\author{
Oreste Floquet \\ Sapienza, Università di Roma \\ Modyco, UMR 7114, Université de Paris Ouest \\ oreste.floquet@uniromal.it \\ orestefloquet@hotmail.com
}

\section{Introduction}

On compte parmi les traits spécifiques de l'aire anglo-normande l'absence de la nasale palatale dans la série des consonnes. Si pour les autres dialectes ancien français la situation demeure assez controversée ${ }^{1}$ du fait que :

La notation des palatales étant de façon générale assez hésitante dans les scriptae,

l'interprétation des graphies est plus problématique (Wüest $1995: 306$ )

pour l'anglo-normand, en revanche, les jugements sont plus nets et ont été récemment réaffirmés de manière on ne peut plus claire. Puisqu'on trouve des rimes en $<\mathrm{n}>$ (que l'on fait généralement correspondre à [n]) s'alternant à des rimes en $<\mathrm{gn}>^{2}$ (que l'on devrait faire correspondre à [n] en position interne et finale), et qu'on les considère comme étant toujours parfaites sous l'aspect phonétique, on en déduit que la nasale palatale a disparu, ou presque, dans n'importe quelle position : c'est le cas, par exemple, de la rime moine : essoigne. Pope (1952: 450) précise qu'à l'origine de la disparition de [n] il faudrait voir une interférence de l'anglais médiéval :

The absence of the palatal sounds $[\lambda]$ and $[n]$ in the English sound-system led to early modification of these sounds and they are ordinarily resolved into dental [1] and [n] preceded by a palatal glide.

bien que ce processus se soit produit de manière graduelle :

[...] but the pronunciation appears to have varied for some time, if one may judge from the practice of individual writers.

De même Burgess (1995 : 343):

Der Palatal-Nasal (geschrieben gn) tendiert auch zur Entpalatalisierung (cf. Reim plein : desdeign, Brendan, 235-236) [...].

Short (2007 : 40) est plus radical, qui considère que la dépalatalisation est déjà parfaitement accomplie dès les plus anciens textes :

The non-palatalisation of 1 (as in fille) and of $n$ (as in seigneur), permitting rhymes such as merveilles : esteiles, feignent : peinent, and the humorous confusion of agnel 'lamb' and anel 'ass'. 
Et encore plus bas :

In parallel to the AN development of palatal $l[\ldots]$, evidence points to palatal $n$ not being distinguished from its dental counterpart: femme : regne ${ }^{3}$ Comput. 469 (the same rhyme in Gaimar 2528, 3595), vaine : bargai[g]ne Comput. 2005, semaine : cumpai[g]ne Brendan. 591, fin : lin Brendan. 43, moine : essoigne Beneit's Becket. 1790. [Short $2007: 100]$

Qu'en est-il du niveau phonologique? Deux possibilités semblent émerger, encore que les auteurs ne tranchent jamais de manière très claire sur ce point : (a) ou bien [n] est devenu un allophone de $/ \mathrm{n} /$; (b) ou bien $[n]$ est un phone inexistant en anglo-normand dans n'importe quelle position. Reste que dans les deux cas on envisage un processus de déphonologisation qui aurait fait disparaitre la nasale palatale de l'inventaire phonémique de l'anglo-normand. Sans nier que la démouillure de $[\mathrm{n}]$ est un phénomène phonétique qui commence à se répandre assez tôt, c'est le raisonnement visant à faire l'économie de /n/ qui nous paraît caduc puisqu'il se fonde exclusivement sur les rimes et sur une étude sommaire de la scripta. Nous allons partir d'une analyse graphique et métrique visant à éclairer les données pour ensuite essayer de développer une intuition de Lote (1955: 237) qui, après avoir constaté que le phénomène des rimes alternant la nasale palatale à la nasale dentale est bien représenté dans tout le territoire, conclut que :

Chez beaucoup de poètes, ces rimes peuvent d'ailleurs être considérées comme des rimes imparfaites ou d'emprunt.

Le but de notre intervention est donc de montrer qu'il n'y a pas lieu de faire l'économie de /n/en anglonormand bien que son rendement fonctionnel demeure assez faible.

\section{Aspects graphiques}

Outil d'investigation privilégié dans les études de phonologie diachronique, l'étude graphématique est une ressource indispensable, encore qu'elle risque d'être insuffisante si on ne la mène pas de manière contrastive; ce qui veut dire qu'il faut se focaliser sur la distribution des graphèmes et donc, pour ce qui nous concerne, sur $<\mathrm{gn}>$ et son concurrent $<\mathrm{n}>$. Comme nous allons le montrer, dans bien de cas l'incertitude règne, si bien que la thèse de Gossen $(1968: 2)$ selon laquelle :

Dans beaucoup de cas, le graphème ne sera qu'une approximation.

se trouve tout à fait confirmée.

Dans une approche autonomiste de la graphie, qui est aussi la nôtre, où on établit une relation de parallélisme entre graphème et phonème et non pas de dépendance :

les méthodes structurales classiques - utilisés en phonologie - peuvent être appliquées aux énoncés écrits [...]. (Anis et alii $1988: 86$ )

puisqu' il faut :

partir des lettres pour arriver aux sons en non l'inverse. (Anis et alii 1988 : 151)

Ainsi, en partant de l'idée que le code graphique et le code oral fonctionnent indépendamment l'un de l'autre, bien qu'ils présentent un certain nombre de convergences (d'ordre phonologique notamment), on a mis en opposition les deux graphes, $<$ gn $>$ et $<\mathrm{n}>$, de façon à comprendre s'ils correspondent à un seul 
graphème ou bien à deux. Le classement que l'on propose se fonde sur la totalité du corpus $\mathrm{ANH}^{4}$, sans distinctions ultérieures selon le temps, l'espace ou la typologie textuelle. On est bien conscient des risques qu'implique une telle démarche du moment qu'elle se dispense d'une analyse fine de la variation, d'autant plus que le débat sur le statut linguistique et socio-linguistique de l'anglo-normand est encore largement ouvert ${ }^{5}$. Et cependant, il nous a paru préférable dans un premier temps d'essayer de dégager des régularités fortes et hautement génériques pour qu'ensuite on puisse mener à bien une étude davantage détaillée. Dans bon nombre de cas, $<$ gn $>$ et $<$ n $>$ semblent se comporter comme deux allographes d'un seul graphème, dont la forme sous-jacente pourrait être $/<n>/$. Parce qu'il n'y a pas de graphies exclusives pour la nasale palatale ou la nasale dentale et qu'en revanche nous avons autant des cas ou $<$ gn $>$ et $<\mathrm{n}>$ pourraient représenter l'une ou l'autre, on peut dire qu'ils semblent commuter librement. Après tout, il semblerait qu'on touche là à la démonstration que ces deux graphes, ayant perdu toute valeur distinctive, ne font référence désormais qu'à un seul phonème, la nasale dentale :

$\{<\mathrm{gn}>,<\mathrm{n}>\} \quad>\quad \mid<\mathrm{n}>/ \quad[\mathrm{n}]$

Passons tout de suite aux exemples concrets. Comme il figure à la rime, le couple moine : essoigne est cité par Short (2007) en tant que prototype de la dépalatalisation et de la perte de toute référence à un son palatal dans $<\mathrm{gn}>$. Toutefois il faut souligner que dans le corpus ANH non seulement essoigne mais aussi moine ne présentent jamais de graphies stables, ce qui est d'autant plus remarquable puisque pour ce dernier il n'y a pas lieu de supposer une ancienne prononciation avec nasale palatale. La forme $<\mathrm{e}(\mathrm{s}) \operatorname{soin}(\mathrm{e})>$ se trouve dans :

La Vie de Saint Thomas Becket, Britton, Annales Monasterii de Burton, Corset, Foedera, Liber Custumarum, Protheselaus, Les Proverbes de Salemon. La Petite Philosophie ; la forme <e(s)soigne> dans: Corset, Foedera, Liber Albus, Apocalypse, Britton, Brut, The Statutes of the Realm. La forme $<$ moine> se trouve dans : La Vie de Saint Thomas Becket, St Brendan, Brut, Les Gius Partiz des Eschez, 'Folie Tristan', L'Estoire des Engleis, The Romance of Horn, La Lumere as Lais, Protheselaus, La Estoire de Seint Aedward le Rei, St. Modwenna; la forme <moigne> dans : Britton, La Destructioun de Rome, Foedera, Anglo-Norman Letters and Petitions and Petitions from All Souls, Manières de Langage, Ancient Petitions relating to Northumberland, Le Livere de Reis De Brittanie e Le Livere de Reis de Engletere. Cette même alternance graphique se répète pour d'autres lexèmes, contenant ou pas une nasale palatale originaire. Prenons le cas des alternances $<$ fontaine $>\sim<$ fontaigne $>$ et de $<$ montaine $>\sim$ $<$ montaigne $>$. La forme $<$ fontaine $>$ se trouve dans : St Brendan, Le livre des psaumes, La Lumere as Lais, La Petite Philosophie, Les Proverbes de Salemon, La Estoire de Seint Aedward le Rei, St. Modwenna, Le Roman de toute Chevalerie, York Memorandum Book. La forme <fontaigne> dans: Apocalypse, Croniques de London depuis l'an 44 Hen III jusqu'à l'an 17 Edw III, Liber Albus, La Destructioun de Rome, Fouke le Fitz Waryn, Anglo-Norman Letters and Petitions and Petitions from All Souls, Le livre de seyntz medicines, La petite Philosophie. la forme $<$ montaine $>$ dans : St Brendan, St. Modwenna ; la forme $<$ montaigne> dans : Apocalypse, The Anonimalle Chronicle, Foedera, Fouke le Fitz Waryn, The Crusade and Death of Richard I, Royal and Other Historical Letters Illustrative of the reign of Henry III. De plus, le choix et le maintien de l'une des deux graphies (le graphème $<\mathrm{n}>$ ou bien le graphème $<$ gn $>$ ) tout le long du même ouvrage, nous semble être une tendance majeure, (encore qu'il faudrait pouvoir la vérifier de manière plus détaillée). Nous en donnons quelques exemples choisis. Dans le St Brendan nous trouvons une majorité de formes en $<\mathrm{n}>$ : $<$ moine $>$, $<$ fontaine $>$ et $<$ montaine $>$. Dans l'Apocalypse, en revanche, une majorité de formes en $<$ gn $>$ : <essoigne $>$, $<$ funtaigne $>$ et $<$ montaigne $>$. Mais parfois on constate un flottement graphique dans le même ouvrage. La forme <essoine $>$ alterne avec <essoigne $>$ dans Britton, Corset et Foedera. On ajoute $<$ Bretaine $>\sim<$ Bretaigne $>$ dans l'Estoire des Engleis (toujours à la rime) et $<$ funtaine $>\sim<$ funtaigne $>$ dans la Petite Philosophie. Finalement, si la dépalatalisation était déjà achevée, on se demande pourquoi $<$ n $>$ n'a pas systématiquement supplanté $<\mathrm{gn}>$ qui, de son côté, se trouve là où il n'a pas de raisons historiques d'apparaître. Par ailleurs, plusieurs indices montrent que (1) ne décrit pas tous les 
contextes possibles. Citons, par exemple, un quatrain du Saint-Modwenna, qui nous sert à démontrer que $/<\mathrm{n}>/$ n'est pas forcément [n] :

(2)

Lessez la veie devers la plaigne
Si vus venez en la muntaine.
Jo voil, ke *Kevin i [se] baigne.'
Sun bastun prent e pus [la] seigne.

1380

Une seule fin de vers en $<$ n $>$ se glisse à l'intérieur d'une suite en $<$ gn $>$. S'agit-il de rimes en [n] ou bien de rimes en [n] ? Le fait que les nombreux quatrains du Saint-Modwenna sont régulièrement bâtis sur quatre rimes identiques nous fait pencher pour la deuxième réponse. Il nous paraît vraisemblable qu'on a affaire à des terminaisons en $[\mathrm{n}]$ pouvant être graphiées de manière différente ${ }^{6}$. Et d'ailleurs, il se trouve que dans ce poème $<\mathrm{n}>$ est la seule forme possible pour montagne. De toute évidence ce mot s'écrit d'une seule manière, quelle que soit sa substance phonique; cela signifie que les graphies ont tendance à se lexicaliser (c'est-à-dire à maintenir une forme graphique spécifique en dépit des évolutions phonétiques) de sorte qu'à nouveau il faut nuancer le caractère général de (1), chaque mot pouvant avoir sa propre histoire graphique $^{7}$. Il y a d'autres contextes où l'opposition graphique $<$ n $>\sim<$ gn $>$ est nette et tout à fait fonctionnelle puisqu'elle fait référence au sens. Un passage de l'Apocalypse nous semble éclairant. La trompette céleste est graphiée de manière régulière $<$ busine $>$, alors que non seulement dans d'autres textes on la trouve graphiée $<$ busigne $>$, mais que dans l'Apocalypse la tendance est sans conteste à la proéminence de $<$ gn $>$ sur $<$ n $>$. Une seule occurrence de $<$ busigne $>$ se réfère au mot besoin, ce qui veut dire que dans ce cas précis la graphie est distinctive. Par ailleurs, cela implique que $<$ busigne $>$ ne peut être prononcé qu'avec une nasale palatale, ce qui d'ailleurs évite de briser la structure à rimes plates. Le choix graphique est donc très conséquent car on évite savamment d'écrire de la même manière les deux mots. Il en résulte que l'on a affaire à deux nasales palatales pour la rime aloigne : busigne, et à deux nasales dentales pour la rime busine : lune $e^{8}$ :

\footnotetext{
E les unt fet ameres cume aloigne,

Dunt perissent par errur ki ne entendent la busigne.

E li quart aungele suna sa busine,

E la tierce par del solail e de la lune
}

1576

On pourrait avoir donné l'impression que les contextes distinctifs ne sont que locaux et marginaux. Mettons en vis-à-vis de <agnel> la forme <anel> qui pour Short (2007: 40) lui serait homophone. Or les deux graphies ne se superposent jamais, la première forme étant la seule à pouvoir indiquer l'agneau?

De tout cela il en ressort une première tendance (qui reste à vérifier en prenant en considération un nombre plus vaste d'items). Que l'on compare ces derniers résultats avec ceux de $<$ funtaine $>$ ou de $<$ muntaine $>$ : l'interchangeabilité entre $<\mathrm{gn}>$ et $<\mathrm{n}>$ n'est possible que là où on ne touche pas aux distinctions sémantiques alors que le contraste est tendanciellement maintenu s'il y a opposition phonémique $/ \mathrm{n} / \sim / \mathrm{n} /$. Cela dit, pour ce qui concerne les verbes à alternance de base, la situation n'est pas toujours très claire à cause des phénomènes de réfection analogique ${ }^{10}$. On trouve des oppositions assez nettes, quoique très faiblement représentatives, comme <venons $>$ (seulement indicatif) et <veignons $>$ (seulement subjonctif) et des oscillations graphiques comme $<$ dones $>\sim<$ doignes $>$ où les informations modales perdent toute leur transparence graphique. On est donc dans un micro-système morphologique où la neutralisation et le contraste graphiques cohabitent; cela nécessite de plus amples enquêtes que nous sommes d'ailleurs en train de poursuivre. En conclusion, à la différence des analyses qui prennent en compte de façon isolée la graphie $<$ gn> comme, par exemple, celle qui semble être implicite dans les affirmations de Zink (1986 : 100), pour qui : 
Quant à $[n]$, il demeure jusqu'à nos jours entre voyelles et ne se dépalatalise complètement en finale que vers le début du XIIe siècle (à en juger par l'abandon de la graphie -ng). (Zink 1986: 100)

et qui sont, à nos yeux, largement insuffisantes, une approche contrastive, permet de mettre au jour un certain nombre de contextes où $/ \mathrm{n} /$ est sans doute encore un phonème de l'anglo-normand.

\section{Aspects métriques}

Venons-en au système rimique. La première question qui se pose est de savoir comment interpréter les rimes du type regne: femme. S'agit-il de rimes parfaites ou de rimes imparfaites? La difficulté réside dans le fait que d'un point de vue purement qualitatif, les deux réponses sont acceptables au même degré. Néanmoins, il nous semble que si on intègre aussi l'aspect quantitatif, il est possible d'entrevoir une solution différente. Lorsqu'on interroge le concordancier $\mathrm{ANH}$, on constate aisément la situation suivante :

(4)

regne

nombre total d'occurrence (textes en prose et textes en vers) : 1438

nombre d'occurrence à la rime ${ }^{11}: 9$

Gaimar, l'Estoire des Engleis

Cum ercevesque tint le sied.

Li rei[s] Pentan dunc reçut regne,

Tut le rëalme de Marcenne.

Wilaf repurcaçad le regne,

Si refud reis desur Mercenne.

Ses dous fiz reçorent le regne

Qu'il ot de sa premiere femme.

Un riches hom maneit el regne,

Bien sai que morte esteit sa femme,

Hue de Rotelande, Protheselaus

D'altre part plus sui gentil femme

Que tel reïne que tent regne;

6616

En nul empirë, en nul regne

Fors Medea si bele femme.

9880

Il n'ad el mund ne en nul regne

Si vil garce ne si vil femme

10160

Que unques el munt en nul regne

$\mathrm{Ne}$ vit des oilz si bele femme,

Philippe de Thaon, Comput 
Le jusdi li dunerent.

E Venus, une femme

$\mathrm{Ki}$ esteit de lur regne

Il est frappant de constater que regne est très peu exploité à la rime et qu'il n'alterne qu'avec deux mots (sept fois avec femme et deux fois avec Mercenne) exclusivement dans trois ouvrages. A-t-on un nombre suffisant d'éléments pour affirmer que <regne> serait désormais dépalatalisé ? De plus, il nous paraît important de souligner le fait qu'on le retrouve toujours dans des modules à rimes plates, alors qu'une suite de plusieurs rimes en $[\mathrm{n}$ ] où se glisserait une rime en $<$ regne $>$ serait un argument certainement plus solide. Par voie de conséquence, rien n'empêche, nous semble-t-il, d'interpréter regne: femme comme une rime figée, un événement très réduit et local donc, qu'on aurait tort d'utiliser pour argumenter en faveur d'un changement plus vaste d'ordre systémique tel que la perte d'un phonème. Nous allons passer en revue d'autres cas du même genre; certains d'entre eux sont cités dans les manuels sous prétexte qu'ils montreraient que la démouillure s'est accomplie :

\section{desdei $(g) n$}

nombre total d'occurrence (textes en prose et textes en vers) $: 5$ nombre d'occurrence à la rime : 3

\section{$\underline{\text { St-Brendan }}$}

Un meis sanz vent nagerent tut plein

Tuit li frere par nul desdeign.

Apocalypse

Ne lur oeveres fet en vein

Par orguil ne par desdein

$\underline{\text { Le secré des secrez }}$

Ke nel faz mie en desdein,

Einz est l'encheisun, seez certein

compaigne $^{12}$

nombre total d'occurrence (textes en prose et textes en vers) : 290 nombre d'occurrence de compaigne à la rime : 8

Fabliaux, (de .iii. dames qui troverent .i. vit)

Veiez si une moie compaigne

Qe doner ma part ne me deygne

$\underline{\text { St-Brendan }}$

Dous feiz tuz dis la semaine

Cil revisdout la cumpaine.

Gaimar, l'Estoire des Engleis

Cil de Seisuine e d'Alemaine

S'ajustoent a lur cunpaine;

Mult fud doté partut Bretaine

Kar tuz jurz cresseit sa cumpaigne. 
Ocis i fud en la champaine,

Poi eschapad de sa cumpaine.

Li cuens Alains, cil de Bretaine,

Bien [i] ferit od sa cumpaigne;

The Hospitallers' Riwle

Mes aut avant, si se [cum]pleigne

Al majur de la cumpaigne,

$\underline{\text { Hue de Rotelande, Protheselaus }}$

Kar les plusors de lor cumpaigne

Sunt ja morz e pris en la plaigne.

Alemai(g)ne

nombre total d'occurrence (textes en prose et textes en vers) : 36 nombre d'occurrence de alemai(g)ne à la rime : 1

Gaimar, l'Estoire des Engleis

Cil de Seisuine e d'Alemaine

S'ajustoent a lur cunpaine;

\section{feignent}

Nombre total d'occurrence (textes en prose et textes en vers) $: 10$ Nombre d'occurrence à la rime : $2^{13}$

\section{St-Brendan}

Pur le bon vent ne s'en feignent,

Mais de nager mult se peinent;

E nepurtant ne s'en feignent:

Mais cum plus vunt, plus se peinent,

essoigne $^{14}$

nombre total d'occurrence (textes en prose et textes en vers) : 75

nombre d'occurrence à la rime : 4

\section{Apocalypse}

Li secund aungeles ki dist tut saunz essoigne,

'Chaet est, chaet est, la grant Babiloine,

\section{$\underline{\text { Robert le Chapelain, Corset }}$}

Les angeles en trai a testemoine

Et quantke Dieux fist saunz essoine

Et quant il le fount par busigne,

Vers Dieu en ount mult grant esoigne

$\underline{\text { La Petite Philosophie }}$ 
Outre la faible quantité de ce type de mots-rime (ce qui est tout à fait remarquable puisqu'ils pourraient créer des équivalences $-\mathrm{s}$ 'ils étaient déjà dépalatalisés- avec des finales à nasale dentale), on notera qu'il existe bien des cas où la dépalatalisation est fort improbable, comme compaigne : deygne dans le fabliau des trois dames, seignent: pei(g)nent, deux fois dans le Comput, ou bien busigne: essoigne dans le Corset. On pourrait soutenir qu'il s'agit de graphies erronées, mais cela rendrait parfaitement circulaire (et contre-factuel) le raisonnement puisqu'on nierait toute possibilité de falsifier l'hypothèse de la disparition précoce de la nasale palatale. Bien au contraire, de telles rimes sont pour nous une autre pièce de la mosaïque puisqu'elles témoignent de l'existence de rimes parfaites en nasale palatale, ce qui rend plus faible l'hypothèse d'une déphonologisation complète. Venons-en à la deuxième question. Nous avons déjà montré les a priori concernant les rimes en $<\mathrm{n}>$ et en $<\mathrm{gn}>$. Or, un principe de cohérence imposerait, du moins à nos yeux, d'appliquer le même raisonnement à $<\mathrm{m}>$ aussi. Bizarrement, l'alternance de rimes à nasale dentale et labiale, ne semble pas remettre en cause l'existence des deux phonèmes $/ \mathrm{n} /$ et $/ \mathrm{m} /$. Nul n'avance d'ailleurs la possibilité d'un seul et unique phonème $/ \mathrm{n} /(\mathrm{ou} / \mathrm{m} /)$ qui serait sous-jacent à $[\mathrm{n}]$ et $[\mathrm{m}]$. On se borne juste à signaler que :

Postvocalic $-m$ and $-n$ can rhyme together: raisun : veium Comput. 209, achaisun : um Comput. 727, peisun : eisum Brendan. 833, garçon : non (< NOMINE) Beneit's Becket. 1351, feim : pain SetDorm. 1074. The equivalence is found in feminine rhyme in PStEdmund. cleime : certeine 143. Graphies are, accordingly, interchangeable: menbres, emfant, etc. (Short $2007: 100$ )

Lote $(1955: 235)$ semblerait avoir compris une telle difficulté lorsqu'il affirme de manière très nuancée que pour l'ensemble du français médiéval :

Ce qu'il y a de remarquable, c'est que $m$ et $n$ après voyelle tonique sont confondues à la rime.

Nous avançons deux hypothèses alternatives qui ne font pas l'économie de /n/. On peut considérer que /n/, $/ \mathrm{m} /$ et $/ \mathrm{n} /$ sont trois phonèmes distincts qui se neutralisent à la rime, le trait pertinent en commun à la base de l'équivalence étant seulement [+ nasal]. Il existe d'ailleurs une tendance morphophonologique à la neutralisation des nasales en fin de mot qui a été décrite depuis longtemps par Walker (1981: 69), et qui pourrait bien être à l'origine de ce type de phénomène :

Nasal neutralization is a transient rule in any case, because of the effects of analogy as well as the loss of nasal consonants in final position subsequent to vowel nasalization.

Cependant, on ne trouve pas d'équivalences $/ \mathrm{m} /: / \mathrm{n} /$ (excepté femme $:$ regne), mais seulement $/ \mathrm{n} /: / \mathrm{m} / \mathrm{ou} / \mathrm{n} /$ : $/ \mathrm{n} /$. Cette lacune nous invite à chercher une solution différente. On propose que pour les rimes $/ \mathrm{n} /: / \mathrm{m} / \mathrm{le}$ trait pertinent soit seulement [ + nasal], alors que pour les rimes $/ \mathrm{n} /: / \mathrm{n} /$ les traits pertinents soient $[+$ nasal] et $[+$ coronal $]^{15}$. Toutefois, il faut éviter qu'une telle solution demeure complètement ad hoc. Or, il se trouve que la coronalité nous permet de fournir une explication à un certain nombre de rimes manifestement approximatives, comme $/ \mathrm{n} /: / 1 /, / \mathrm{r} /: / 1 /, / \theta /: / \delta /$, sur lesquelles généralement on glisse quant aux conséquences phonologiques qu'elles entraineraient si on appliquait le même raisonnement qu'on utilise pour /n/ :

Philippe de Thaon, Comput 
E uitovre e decembre

Altretant ensemble.

Mais qui ben entendrat

E sutilement i verrad.
1138

1466

L'avantage d'une telle hypothèse est d'être assez restrictive puisqu'elle ramène toutes ces rimes à un seul constituant phonétique : le noeud coronal ${ }^{16}$. Reste à comprendre pourquoi à l'intérieur de cette classe un certain nombre d'appariements ne sont pas possibles (par exemple $/ \mathrm{n} /: / \mathrm{r} / \mathrm{ou} / \mathrm{n} /: / 1 /$ ). C'est parce que dans ces rimes approximatives la coronalité est une condition nécessaire, mais non suffisante : un deuxième trait doit entrer obligatoirement en jeu : la nasalité, l'antériorité, la continuité ou la répartition ${ }^{17}$.

(graphique 1)

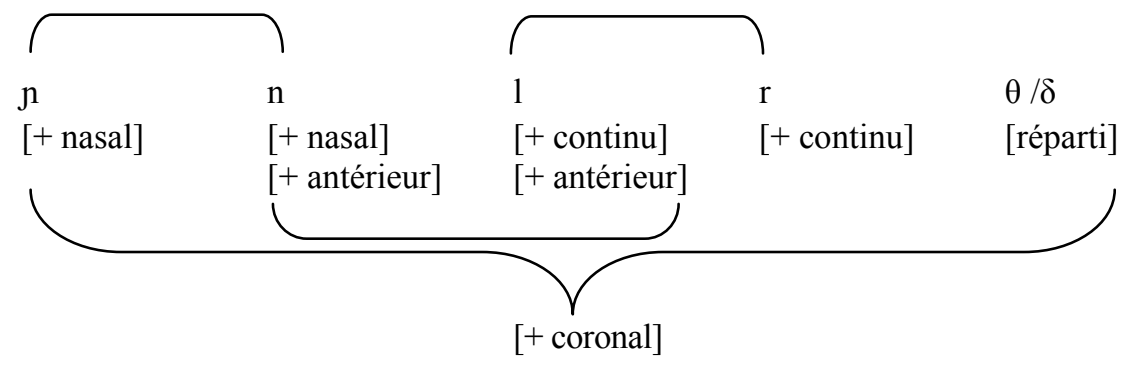

De la même façon, on pourrait tenter d'expliquer le fonctionnement des imperfections des rimes diphtongales /aj/ : /ej/ et /oj/ : /ej/, où /j/ est sans conteste [+ coronal]:

\section{Philippe de Thaon, Comput}

Marz est li primers mais

Que Romulus li reis

2916

Apocalypse

E çoe est la premere voiz ke il oi

Cume de busine parlante a sei.
686

encore que cela mérite de plus amples recherches ${ }^{18}$.

\section{Considérations finales}

Un paradoxe hante depuis toujours la versification anglo-normande. C'est un fait notoire que d'une part il s'agit d'une versification irrégulière par rapport à la versification continentale puisque le nombre syllabique et la césure sont parfois flottants ; d'autre part depuis Vising (1884) on s'accorde, en revanche, pour considérer le système rimique tout à fait régulier :

Ce qu'il y a de plus frappant et de plus populaire dans la versification française, c'était la rime. Aussi la gardait-on pure en Angleterre, tandis que le nombre des syllabes et la césure étaient négligés. (Vising $1884: 64$ ) 
Sous prétexte que la rime est foncièrement parfaite du point de vue phonétique, on en a déduit que les irrégularités qui émergent ne sont que des phénomènes soit apparents (puisqu'on suppose que les graphies divergentes ne font que cacher une réalité phonétique identique), soit fautifs (c'est-à-dire liés à des usages aberrants et minoritaires), soit superficiels (si on suppose une intervention tardive des copistes). A partir de l'axiome de la rime pure, les philologues et les linguistes ont été amenés à ne pas considérer les irrégularités comme telles, mais plutôt à les ramener à un structure sous-jacente phonétiquement régulière; d'où les inférences sur la disparition de la nasale palatale - tout à fait légitimes, après tout - qui ont été l'objet de notre discussion jusqu'à présent. Partir d'un axiome complètement différent signifie soutenir l'hypothèse que les appariements rimiques se font tour à tour de manière plus ou moins approchée en fonction du nombre de traits pertinents qui y sont impliqués. Les équivalences se distribuent le long d'un continuum qui va d'une cohésion minimale, où les séquences terminales partagent au moins un $\operatorname{trait}^{19}$ (comme dans le cas des rimes $/ \mathrm{n} /: / \mathrm{m} /$ ), à une cohésion maximale, où les séquences terminales possèdent tous les traits en commun (ce qui correspond à la rime parfaite). Il s'ensuit que l'on peut aisément éviter de scinder la versification insulaire en deux parties, l'une irrégulière, l'autre régulière et les ramener, au contraire, à une tendance commune puisque l'approximation s'appliquerait tant au mètre qu'aux rimes. Comme elle implique qu'une certaine dose de sous-détermination phonologique soit implicite au niveau métrique (puisque les segments ne sont pas complètement identiques entre eux), notre représentation pourrait paraître tout à fait $a d h o c$, alors qu'il s'agit d'une caractéristique que l'on retrouve ailleurs, par exemple en métrique orale, française, italienne et anglaise ${ }^{20}$. Floquet (2007), (2008) et (2009) analyse la structure formelle des comptines où l'approximation, qu'elle soit métrique (puisqu'on constate un anisosyllabisme assez répandu dans tout le corpus aussi bien qu'un patron accentuel facilement déformable), textuelle (vu la précarité des superstructures métriques), ou rimique, représente sans doute la caractéristique majeure qui les différencie des textes littéraires (dont les frontières entre les vers, les formes strophiques et les rimes sont toujours suffisamment bien définies). La sous-détermination ne saurait être un obstacle à l'intercompréhension, puisque, comme l'a montré Laks (2007), elle est constamment supplée par d'autres paramètres, en l'occurrence le mètre :

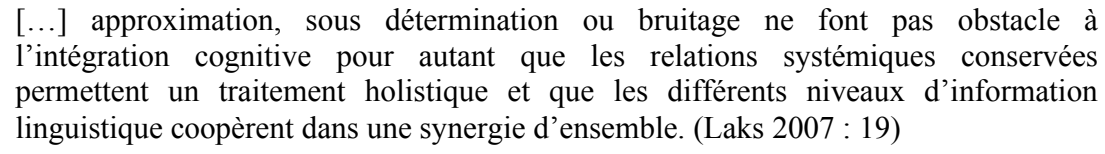

Pour ce qui est des rimes des comptines françaises, Floquet (2009) observe que la coronalité est le commun dénominateur de la plupart des rimes approximatives : $[\mathrm{s}]:[\mathrm{z}],[\mathrm{s}]:[\mathrm{S}],[\mathrm{z}]:[3],[5]:[3],[\mathrm{t}]:[\mathrm{S}]$, [t] : [3], ainsi que [n] : [n]. Or, puisqu'il est tout à fait absurde de supposer qu'en raison des rimes du type $[n]:[n]$ (ou bien $[\tilde{\varepsilon}]:[\varepsilon]$, car Floquet (2009) étudie aussi les imperfections concernant les voyelles nasales), la nasale palatale et les voyelles nasales n'existent pas en français contemporain, force est d'admettre qu'une équivalence rimique, à elle seule, n'est pas toujours un indice fiable de la structure d'une langue, la métrique pouvant être autonome par rapport à la phonologie.

Pour conclure, le système de l'approximation anglo-normande et sa ressemblance avec les métriques orales, outre le fait de remettre en question la soi-disant démouillure de $/ \mathrm{n} /$, ce qui était somme toute notre but, nous suggère aussi - du moins à nos yeux- de reconsidérer la versification insulaire sous un angle différent. Il reste à comprendre, à condition que l'on maintienne notre hypothèse, quelle est la part de l'oral et de l'écrit dans un système qui tolère de telles erreurs qu'on ne trouve pas sur le continent.

\section{Références bibliographiques}

Anis, J., Chiss, J.-L., Puech, Ch. (1988). L'écriture, théories et descriptions. Bruxelles : De Boeck.

Andrieux, N., Baumgartner, E. (1983). Systèmes morphologiques de l'ancien français. Bordeaux : Sobodi.

Boltanski, J.-E. (1999). Nouvelles directions en phonologie. Paris : PUF. 
Burgess, G., S. (1995). Französische Skriptaformen IV - England. In Holtus G. , Metzeltin M., Schmitt Ch., (éds), Lexicon der Romanistischen Linguistik, II, 2, Tübingen : Max Niemeyer Verlag, 337-346.

Chaussée, F. de la (1989). Initiation à la phonétique historique de l'ancien français. Paris : Klincksieck.

Clements, G.N. et Hume E. (1995). The Internal Organization of Speech Sounds. In J. A. Goldsmith, The Handbook of Phonological Theory, (éd.), Cambridge : Blackwell Publishers, 245-306.

Floquet, O., (2007). Preliminari sulla fonologia della rima nelle filastrocche : aspetti descrittivi ed interpretativi. Strumenti Critici, 1, 1-47.

Floquet, O., (2008). Une hypothèse connexionniste sur la métrique orale. Cognitive Philology, 2, http://padis2.uniroma1.it:81/ojs/index.php/cogphil

Floquet, O., (2009). De quelques aspects de la rime dans les comptines françaises. Quaderni del Laboratorio di Linguistica della Scuola Normale Superiore, vol 8, http://alphalinguistica.sns.it/Quaderni.htm.

Einhorn, E. (1974). Old French a concise handbook. London : Cambridge University Press.

Fouché, P. (1961). Phonétique historique du français, les consonnes. Paris : Klincksieck.

Gossen, Ch.-Th. (1968).Graphème et phonème : le problème central de l'étude des langues écrites du Moyen âge. Revue de Linguistique romane, XXXII, 1-16.

Hall, T.A., (1997). The Phonology of Coronals. CILT 149, Amsterdam/Philadelphia : John Benjamins, Publishing Company.

Jong, Th. de (1988). L'Anglo-normand du $13^{\mathrm{e}}$ siècle. In van Reenen P., van Reenen-Stein K., (éds), Distribution spatiales et temporelles, constellations des manuscrits (études de variation linguistique offertes à Anthonij Dees à l'occasion de son 60e anniversaire), Amsterdam/Philadelphia : John Benjamins Publishing Company, 103-112

Kibbee, D.A. (2000). Historical perspectives on the place of anglo-norman in the history of the French language. French Studies, 137-153.

Laks, B. (2007). De l'approximation dans la relation phonétique / phonologie. In Castagne E., (éd.), Les enjeux de l'intercompréhension (Stakes of intercomprehension), Reims : Presses de l'université de Reims, 181-201.

Lote, G. (1955). Histoire du vers français. t.3, Paris : Hatier.

Rothwell, W. (1975). Anglo-norman Perspectives. The Modern Language Review, 41-49.

Rothwell W. (2004). Ignorant scribe and learned editor : Patterns of textual error in editions of Anglo-French texts. http://www.anglo-norman.net/articlesA/

Shapiro, I. (1984). Sémiotique de la rime. Poétique, 501-519.

Short, I. (2007). Manual of Anglo-Norman. London : Anglo-Norman Text Society.

Trotter, D. (2003). L'anglo-normand : variété insulaire ou variété isolée ? Médiévales, 43-54.

Vising, J. (1884). Sur la versification anglo-normande. Uppsala : R. Almqvist - J. Wiksell éditeurs

Walker,D. C. (1981). Old French Morphophonology. Ottawa : Didier

Wüest, J. (1995). Französische Skriptaformen II - Pikardie, Hennegau, Artois, Flandern. In Holtus G. , Metzeltin M., Schmitt Ch., (éds), Lexicon der Romanistischen Linguistik, II, 2, Tübingen : Max Niemeyer Verlag, 300 -314.

Zink, G. (1986). Phonétique historique du français. Paris : PUF.

Zwicky, A. (1976). His rock and roll has got to stop, on rhyme in rock music. Chicago Linguistic Society, 676-697.

${ }^{1}$ Ce qui fait que l'on constate un flottement dans les datations (pour Fouché (1961: 808-809) la démouillure en position finale serait déjà accomplie au XIe, pour La Chaussé (1989: 74) cette date ne marquerait que le début du procès, pour Zink (1986 : 100) finalement, il faut pousser la dépalatalisation complète jusqu'au XII ${ }^{\mathrm{e}}$ ).

${ }^{2}$ Par la suite, nous allons faire référence seulement à la graphie $<\mathrm{gn}>$, tout en sachant qu'elle résume un éventail plus vaste de réalisations allographiques, et notamment : $<$ ign $>,<$ ng $>,<$ ing $>$, etc. Voir, entre autres, Einhorn $(1974: 4)$.

${ }^{3}$ Pour les détails concernant cette rime voir au chapitre 2. 


\footnotetext{
${ }^{4}$ L'Anglo-Norman Hub est une base de données contenant une grande partie de la production littéraire et non littéraire anglo-normande (www.anglo-norman.net).

${ }^{5}$ Voir, entre autres, Rothwell (1975), Jong (1988), Kibbee (2000) et plus dernièrement Trotter (2003).

${ }^{6}$ Rien n'empêche que la palatale de <plaigne $>$ soit due à une prononciation hypercorrecte. Dans le Protesehlaus $7225-7226$ on le trouve en rime avec $<$ cumpaigne $>$.

${ }^{7}$ Sur la dyscrasie entre phonie et graphie en anglo-normand voir aussi Rothwell (2004).

${ }^{8}$ Dans le Brut aussi les graphies vont de pair, nous semble-t-il, avec la phonie, puisque $<\mathrm{n}>$ réalise [n] et $<$ gn $>$ se réfère à la nasale palatale: $<$ moine $>$, <essoigne $>$, $<$ Bretaigne $>$.

${ }^{9}$ Voici les cinq occurrences de $<$ agnel $>$, auxquelles on ajoutera les douze de $<$ agnels $>$ que l'on omet de peur de n'alourdir excessivement l'argumentation :
}

\section{$\underline{\text { Liber Albus }}$}

Qe nul vende un agnel pluis chier qe pur vi deniers

$\underline{\text { Roman de toute Chevalerie }}$

De quei put home a femme fere compareison?

Plus est simple quant volt qe agnel ne colom,

Plus cointe qe serpent, ardante cum dragon

\section{$\underline{\text { St.-Brendan }}$}

Jo sui li fels qui Deu haï,

Le simple agnel as lus trahi.

$\underline{\text { Nominale sive Verbale }}$

Hurtonur motoun et agnel

Ram wethur and lombe fe.

\section{La Vye de Seynt Fraunceys d'Assise}

Kar Jhesu ke est duz e bel

Est signifyé par le agnel

${ }^{10}$ Pour le système verbal, voir Andrieux et Baumgartner (1983).

${ }^{11}$ On exclut les cas où la forme figure en apparat, ou bien quand il y a eu une intervention de la part de l'éditeur :

\section{Gaimar, l'estoire des Engleis}

[Cinquante treis] anz tint le regne,

[Cristienté] e dreit [baptesme]

Refud dunc mort tuit ensement

Ecbrit [son fiz saisit le regne]

12 Le nombre total comporte toutes les réalisations possibles: compaigne cumpaigne, conpaigne, compaigne, cunpaine, cumpaine, compaine.

${ }^{13}$ Deux autres occurrences se trouvent dans l'apparat du Comput :

\section{Philippe de Thaon, Comput}


978-2-7598-0534-1, Paris, 2010, Institut de Linguistique Française

Phonétique, phonologie et interfaces

DOI $10.1051 / \mathrm{cmlf} / 2010027$

\section{E de Capricornum}

Vëez ore par raisun:

Deus granz choses seignent

Cil qui cest signe peignent:

Chef de chevere devant,

Cüe de serpent grant,

E en mi un nud peinent

Pur quei devise seignent.

[L1767] cornes i feignent [M] [L1772] Que pur devise feignent [M]

${ }^{14}$ Le nombre total comporte toutes les réalisations possibles : essoigne, essoin, esoigne, essoine.

${ }^{15}$ Pour la coronalité de [n], on renvoie aux tableaux de Walker (1981 : 12-14).

${ }^{16}$ Sur la phonologie et la phonétique des coronales, voir Hall (1997). Sur le noeud coronal et plus en général sur la structure des traits distinctifs, notre point de référence est la théorie de la géométrie des traits; voir Clements et Hume (1995) ainsi que Boltanski (1999).

${ }^{17}$ Conformément à la géométrie des traits, [+ coronal] domine [+ antérieur] et [+ réparti].

${ }^{18}$ Puisqu'il pourrait s'agir aussi de rimes parfaites qui seraient masquées par la graphie.

19 A la différence de Shapiro (1984), on considère que tous les traits peuvent participer à la rime, qu'ils soient marqués ou pas.

${ }^{20}$ Pour l'anglais voir Zwicky (1976). 